\title{
Traduire
}

Une autre perspective sur r tr traduction

Revue française de la traduction

$235 \mid 2016$

Luxe, mode... et traduction !

\section{Puisque « nous le valons bien »...}

Helene Tammik

Traducteur : Marie-Céline Georg et Christine Cross

\section{(2) OpenEdition}

Journals

Édition électronique

URL : https://journals.openedition.org/traduire/843

DOI : $10.4000 /$ traduire.843

ISSN : 2272-9992

Éditeur

Société française des traducteurs

Édition imprimée

Date de publication : 1 décembre 2016

Pagination : 42-53

ISSN : 0395-773X

Référence électronique

Helene Tammik, «Puisque « nous le valons bien »... », Traduire [En ligne], 235 | 2016, mis en ligne le 01 décembre 2018, consulté le 07 juillet 2021. URL : http://journals.openedition.org/traduire/843 ; DOI : https://doi.org/10.4000/traduire.843 


\section{Puisque " nous le valons bien "...}

\section{Helene Tammik}

J'adore le luxe. Pas vous?

Le luxe d'une bonne nuit de sommeil, où le réveil ne viendra pas vous imposer un démarrage trop matinal. Le luxe de rentrer, épuisé, d'une dure journée de travail pour trouver le dîner en train de mijoter et la table mise : il ne vous reste plus qu'à vous effondrer dans votre fauteuil préféré, un verre de vin à la main, prêt à décompresser.

La vie nous offre (parfois) de ces moments où nous pouvons savourer le luxe des plaisirs simples. Et puis, il y a l'autre luxe, celui qui revient très cher. Lorsqu'on s'approvisionne auprès des grandes marques, on n'achète pas simplement un produit : on adopte un mode de vie, une certaine façon d'être. Les marques en question vendent un "état d'esprit ", ce sentiment fabuleux de savourer des plaisirs réservés à quelques heureux élus (qui en ont les moyens).

Le métier que j'exerce est celui de traductrice (et de rédactrice) pour le compte de marques ou de "maisons " de luxe - qui, même en anglais, préfèrent être désignées par ces termes français - ce qui me vaut le privilège de passer mes journées plongée dans l'univers des montres à tourbillon suisses, des sacs en crocodile fabriqués à la main, des robes de tulle ornées de perles cousues main et des voitures aux lignes racées, et de me parfaire à l'art de vendre du rêve.

Traduire dans le domaine du luxe constitue un créneau bien particulier, même si celui-ci englobe une large gamme de produits et de prestations. Les principales compétences requises sont une plume enlevée et de bonnes prédispositions plus qu'une connaissance approfondie d'un secteur donné. Dans ce monde, toutes les communications ont, à la base, la même motivation, à savoir vendre, même si ce n'est pas toujours évident de prime abord. Pour saisir les implications de la traduction dans l'univers du luxe, il faut donc commencer par comprendre la psychologie des marques.

Telle marque ne vous vend pas simplement un produit ; la démarche est bien plus complexe et bien plus élaborée que cela : elle vous vend un style de vie, une image de la personne que vous êtes ou que vous rêvez d'être. 
Les différents axes de communication ratissent large, mais ne visent qu'un seul objectif. Le client ne sera jamais fidèle à un produit mais à la marque, à ses valeurs, à ses messages et au style de vie qu'elle incarne.

Un parfum signé de cette marque-là n'est pas qu'un parfum ; c'est un élément essentiel de la personne que vous allez devenir, cet être plus riche, plus sophistiqué et plus fascinant(1).

\section{Qu'est-ce que le luxe?}

Bien entendu, le luxe va généralement de pair avec l'argent, d'une manière ou d'une autre, mais cela ne s'arrête pas là. Pour citer la fameuse petite phrase de Coco Chanel : "Le luxe, ce n'est pas le contraire de la pauvreté mais celui de la vulgarité ".

Autrefois, celui qui entrait dans l'univers du luxe pénétrait dans un monde privilégié, un milieu averti, exclusif et fortuné. Aujourd'hui, grâce aux moyens de communication planétaires et à la hausse du pouvoir d'achat (surtout en Chine), certaines marques haut de gamme souffrent d'une surexposition et de la désaffection des consommateurs.

À titre d'exemple, l'inventeur supposé du trench en gabardine - cette maison spécialisée dans la confection de vêtements d'extérieur, fondée en 1856 - est une marque de luxe qui a connu de réelles difficultés il y a une dizaine d'années en raison de son adoption en masse par la rue(2), ce qui a mis en péril l'identité de la marque. "Lorsqu'une marque devient trop populaire (au sens primitif du terme), elle aura toujours du mal à regagner son cachet, et il faudra probablement compter plus d'une génération pour y parvenir ", estime Rita Clifton, présidente d'Interbrand, société de conseil en stratégie et design de marques. "Les marques de luxe doivent réunir trois facteurs essentiels : un produit fort de fabrication robuste, un vécu et une image de marque solides, mais aussi un public connaisseur visiblement friand du produit en question(3)."

Au final, cette marque a réussi à surmonter la crise, parce que ce petit flottement au niveau de son positionnement sur le marché est essentiellement resté un phénomène britannique, sans répercussions sur son image à l'échelon mondial.

En 2015, le Musée Victoria \& Albert de Londres a proposé une exposition intitulée : "Qu'estce que le luxe?"

(1) Elephate, agence de Content marketing et SEO (site Internet).

(2) Julia Day, Burberry doffs its cap to 'chavs', The Guardian (01/11/2004, UK).

(3) Ibidem. 
Dans la documentation promotionnelle, on pouvait lire : "Le luxe fait l'objet de controverses depuis toujours. Plus récemment, l'essor et l'omniprésence des marques de luxe dans un contexte caractérisé par les inégalités sociales ont donné lieu à des questionnements sur ce que l'on entend par "luxe" de nos jours "(4).

\section{Luxe ou vulgarité ?}

II y a quelques années, j'ai eu l'occasion de traduire le descriptif d'une pochette vendue dans le commerce pour le prix modique de $168000 €$ et qui me semblait fort jolie. Mais posséder de tels objets pose problème. L'objet vous plaît, certes... Mais est-ce un luxe ou est-ce seulement vulgaire? Les autres vous envieront-ils ou se moqueront-ils de vous dans votre dos? Est-ce de bon goût ou de goût douteux ? La certitude à cet égard est un luxe en soi(5).

Heureusement, il existe le " Definery " ou guide du luxe pour vous aider(6). Ainsi, mon tout dernier achat qualifiable de "luxe " a été soumis à l'épreuve du Definery et serait, selon ce dernier, CATÉGORIQUEMENT VULGAIRE.

\section{La différence entre une marque haut de gamme et une marque de luxe}

Une marque haut de gamme ou " premium " est celle qui se vend à un prix plus élevé - qu'elle doive son renom à une campagne publicitaire qui fait mouche ou au parrainage d'un people-, mais qui ne repose pas sur une excellence intrinsèque et dont le succès peut être fugace. Car le luxe n'est pas qu'une question de prix.

Pour posséder ce que Vadim Grigorian et Francine Espinoza Petersen(7) appellent la " légitimité du luxe ", une marque doit associer un processus de production exceptionnel à un produit de la plus grande qualité capable d'éveiller un lien émotionnel chez l'acheteur, et à une solide tradition ou une histoire distinctive.

(4) Exposition proposée par le Musée Victoria \& Albert (V\&A) du 25 avril au 27 septembre 2015 www.vam.ac.uk/content/exhibitions/what-is-luxury/what-is-luxury-about-the-exhibition/

(5) Traduction d'une citation prise sur le site du V\&A.

(6) http://www.vam.ac.uk/content/exhibitions/what-is-luxury/the-definery/

(7) Vadim Grigorian et Francine Espinoza Petersen, Designing luxury experience, Editors' Pick, Marketing \& Consumers (Strategy \& Management, 21/05/2014). 


\section{Les trois piliers de la " légitimité du luxe "}

\section{Un processus de production exceptionnel}

Outre la parfaite maîtrise de leur discipline, les fabricants de véritables produits de luxe garantissent une précision irréprochable et des finitions sans défaut. Leur profond respect des traditions se double souvent d'une grande capacité d'innovation. La production de luxe représente un investissement en temps. Derrière le produit, on devine une passion, une curiosité quant à la nature de l'objet, des compétences longuement affûtées, une fascination pour les matières et les techniques.

\section{Un lien émotionnel}

Le luxe dépasse les fonctionnalités d'une offre matérielle. II doit toucher les sentiments des clients en leur offrant une valeur symbolique et affective.

Le glamour est un élément émotionnel essentiel du luxe. D'une manière subliminale, il nous fait croire à la possibilité de devenir quelqu'un d'autre, quelqu'un de meilleur dans d'autres circonstances(8), ce désir vif reste toujours en filigrane dans la proposition de la marque.

Ensuite, il y a la relation de l'acheteur avec l'objet lui-même : avec certes le plaisir de posséder un tel objet, mais également celui de le toucher, de découvrir ses qualités insoupçonnées ou surprenantes ; le plaisir de l'utiliser, de laisser vos sens communiquer avec ses matériaux nobles et d'apprécier son design harmonieux et esthétique, son caractère raffiné ; le plaisir du discernement, la perception de l'exclusivité et de la sophistication obtenues grâce à une prise de conscience de son histoire et de sa valeur.

Parfois, ironie du sort, les marques de luxe ne doivent pas miser sur une "qualité impeccable " pour obtenir la valeur symbolique et le pouvoir émotionnel souhaités. Par exemple, alors qu'on pourrait considérer le bruit des grosses voitures de sport italiennes et leur surpuissance inutile comme des "défauts", du point de vue du luxe, ces éléments font bel et bien partie de la philosophie de la marque : dans le luxe, la passion et le rêve sont aussi importants que la fonctionnalité. Les fabricants de ces voitures de prestige dépensent des sommes folles en R\&D pour que les portières se ferment avec un claquement qui fait suffisamment "cher ". Une illusion consentie, semble-t-il, par toutes les parties prenantes.

Une autre émotion exploitée par les marques de luxe est celle des clients qui se considèrent comme méritants et dignes d'une récompense : dans leurs démarches marketing elles essaient souvent de suggérer que leurs produits conféreraient en quelque sorte un pouvoir illimité.

(8) V. Postrel, The Power of Glamour (Simon \& Schuster, 2013). 
Dans un monde de plus en plus dématérialisé, les spécialistes du marketing s'attachent désormais à lier les clients à leur marque par les émotions, en leur proposant des expériences mémorables.

\section{Une tradition ou une histoire liée à la marque}

Une véritable marque de luxe a des racines. Son histoire n'est pas inventée de toutes pièces, mais est le récit de faits véridiques. L'un des aspects positifs de la traduction pour ces grandes maisons est que leur histoire et leurs valeurs servent de trame au dialogue avec leurs clients et avec un public bien plus large. Les marques qui créent de la valeur sur plusieurs générations deviennent un élément vital de notre culture, d'après la spécialiste de la stratégie de marque Rebecca Robins(9).

Cet ancrage dans l'histoire culturelle de la nation constitue un atout précieux pour une marque, et les gardiens actuels de son image (qui font parfois partie de la famille des fondateurs) doivent préserver ce patrimoine avec intelligence pour perpétuer la pertinence de la marque. Ainsi, en se racontant sous forme d'histoire, la marque fait la part belle à son côté humain, elle tisse la trame d'un récit ininterrompu qui forge un lien sentimental avec sa clientèle.

\section{L'évolution du marché du luxe}

Même si les années qui ont suivi le krach financier de 2008 ont vu une renaissance du secteur du luxe, la croissance est désormais en train de fléchir. Selon l'étude mondiale de Bain \& Company sur le marché des produits de luxe, le secteur des biens personnels de luxe pesait 253 milliards d'euros en 2015 et devrait atteindre 280 à 295 milliards d'euros d'ici 2020. Et le terrain des achats de luxe se déplace vers l'Asie : environ la moitié des acquisitions d'articles de luxe sont réalisées par les classes moyennes chinoises, catégorie sociale en pleine expansion.

De plus, les consommateurs de luxe rajeunissent, comme en témoigne un sondage(10) : "Les nouveaux acteurs du marché du luxe ont entre 18 et 34 ans. Ils sont mondialisés, numérisés, optimistes, ouverts à la nouveauté, prompts à recommander et à partager des produits, et sensibles à la durabilité ". Plus de $20 \%$ des milliardaires de la planète ont actuellement entre 45 et 54 ans, d'après un rapport récent(11). Par ailleurs, on constate une conscience

(9) Meta-luxury: Brands and the Culture of Excellence, Rebecca Robins, directrice mondiale d'Interbrand, et Manfredi Ricca (Palgrave Macmillan, 2012).

(10) The True Luxury Global Consumer Insight, sondage réalisé par la fondation Altagamma avec le Boston Consulting Group http://www.luxesf.com/wp-content/uploads/2014/06/BCG-Altagamma-True-Luxury.pdf

(11) Rapport de Camper \& Nicholsons et Wealth-X, cité dans Some Girls are Bigger than Others, Alan Harper (Vanity Fair, septembre 2016). 
environnementale accrue dans ce secteur, avec des clients qui se tournent vers des alternatives plus écologiques et poussent les marques de luxe à réfléchir à la provenance de leurs métaux précieux, de leurs fourrures exotiques, etc.

Alors qu'autrefois, le luxe se définissait souvent par le coût des matériaux et la qualité du savoir-faire nécessaire, le rythme de vie effréné et les communications incessantes de l'époque actuelle ont élevé au rang de luxe des choses moins tangibles, comme le temps pour soi, le confort, l'excitation ou l'aventure. Les consommateurs les plus jeunes, en particulier la génération $Y$, sont saturés de produits de luxe et transforment ce paysage en imposant une transition vers un nouveau concept : dépenser des sommes importantes pour vivre des expériences exceptionnelles (en particulier les voyages). Cette évolution peut du reste s'observer dans l'importance croissante accordée aux " moments uniques " dans les publicités pour des produits de luxe.

\section{En quoi la traduction du luxe est-elle différente?}

Les enjeux sont énormes pour une marque de luxe. Pour asseoir sa position sur la scène mondiale, il faut que toutes ses formes de communication, dans toutes les langues, "parlent " d'une seule voix et expriment une identité monolithique. Leur capacité - ou leur incapacité à obtenir ce résultat aura forcément un impact direct sur la fidélité des clients et donc sur les ventes.

Pour ces marques-là, il est essentiel d'assurer une cohérence dans l'image qu'elles renvoient au public, de véhiculer la même vision et les mêmes promesses dans toutes les langues, cultures et zones géographiques. Une identité de marque doit réussir à englober, sous une forme attirante, des éléments intangibles tels que le désir, l'aspiration, l'ambition et, au bout du compte, la satisfaction, puisque les achats de luxe relèvent autant d'un choix émotionnel que d'un investissement dans un style de vie. Après avoir consacré une fortune à la création d'un profil public dans une langue, une marque qui ne réussit pas à traduire cette identité à l'échelle internationale non seulement va avoir du mal à conquérir de nouveaux marchés, mais risque de porter atteinte à ses positions existantes.

Nous savons tous qu'une erreur de traduction ou un choix malencontreux dans le nom d'un produit affiché sur les réseaux sociaux peut causer un tort colossal à l'image de la marque en question. De l'extérieur, cela peut faire sourire, mais plus la marque est connue, plus elle tombera de haut : l'inévitable attention des médias et l'effet de ridicule liés à son positionnement haut de gamme vont altérer son image aux yeux du public, car le message transmis sera en contradiction avec les concepts de base qu'elle s'est efforcée à grand peine d'associer à son nom : qualité, exclusivité, savoir-faire artisanal et désirabilité. 
Les marques de luxe doivent donc vérifier toutes leurs traductions à la virgule près. Souvent, elles vont travailler avec une équipe de traducteurs fidèles et de confiance, pour peaufiner ensemble un style qui correspondra aux clients dans chaque langue. Les responsables marketing auront pris soin de définir une palette strictement contrôlée d'images et d'ambiances, par exemple " jeune et frais " ou " sensuel et aventureux "; ces notions font partie intégrante de l'identité de la marque, et même lorsqu'elles ne sont pas explicitement citées, toute communication textuelle devra insister sur ces caractéristiques-là.

Certaines grandes marques ont réussi à conserver et à exporter leur positionnement haut de gamme sur tous les marchés notamment grâce à leur identité textuelle forte. Pour ce faire, chacune dispose de sa propre charte éditoriale élaborée par ses équipes de communication.

\section{Charte éditoriale}

La charte éditoriale définit le " terrain de communication " de la marque : les grands sujets et thèmes à aborder, les messages principaux qui expriment et transmettent l'identité de la marque, ses valeurs et sa stratégie. Ces messages enrichissent le discours, déterminent l'approche rédactionnelle de la marque et le traitement des thèmes. La charte éditoriale comporte des directives sur la manière d'intégrer les messages essentiels afin de bâtir une narration incarnant et renforçant l'identité de la marque tout en mettant en lumière ce qui la distingue de ses concurrents. Elle donne également des indications sur le style et le ton à adopter pour exprimer la marque, notamment les règles de langue et de champ lexical.

Pour ce qui me concerne, le texte source ayant été rédigé en portant une attention particulière à la charte éditoriale, il me faut étudier attentivement cette dernière avant de commencer une traduction pour un nouveau client. Je dois entrer dans son " univers " pour m'imprégner des valeurs de la marque et me faire une idée du champ lexical et des domaines conceptuels admis. Par exemple, une maison (ou son agence de communication) peut avoir décrété que les mots pioneer et innovator sont trop forts. Je dois donc me contenter de forerunner.

Certaines expressions que je trouve un peu discordantes et peu anglaises - comme it's in our $\underline{D N A}$, the universe of Brand $X$, Discover our new range, in line with our design codes, beauty $\underline{\text { rituals }}$ - sont devenues monnaie courante dans les textes rédactionnels sur la beauté et la mode. C'est essentiellement dû, il me semble, à l'origine française d'une grande partie de ces marques, et au fait que les rédacteurs non anglophones au sein des services de communication ont intégré ces termes aux accents gaulois dans leur " style officiel ", d'où leur prolifération dans les publicités et les médias britanniques.

\section{Liberté créative}

Même si le style d'écriture et le vocabulaire sont étroitement encadrés, j'ai souvent une certaine liberté pour m'éloigner du texte source, le restructurer en déplaçant des blocs de texte, et 
modifier la hiérarchie des idées. Par exemple, si le principal argument de vente n'est pas le même dans la culture cible, je vais naturellement mettre en avant l'aspect le plus vendeur : dans le cas d'un hôtel, l'accessibilité depuis le Royaume-Uni sera peut-être plus intéressante pour un public britannique que le fait que George Sand y ait séjourné (ou peut-être pas).

Toute trace d'hésitation dans la syntaxe, de maladresse dans les phrases ou d'étrangeté dans les images employées est proscrite. Chaque phrase est créée stratégiquement en faisant appel aux mêmes compétences linguistiques que celles d'un rédacteur pour capter l'imagination, éveiller le désir et contribuer à bâtir le récit de la marque.

\section{Faux pas culturels}

Les attributs utilisables pour décrire un produit dans une langue peuvent être jugés peu pertinents dans une autre. II faut donc parfois changer d'angle d'attaque. Quel meilleur exemple que le concept français de séduction, omniprésent dans les traductions du domaine du luxe? Dans les cultures anglophones, le mot seduction peut avoir des connotations très négatives voire sordides, évoquant la coercition et l'abus. À l'inverse, dans la culture française, la notion de séduction s'utilise pour vendre presque tout, depuis les yaourts jusqu'aux prouesses des hommes politiques, pour paraphraser la journaliste Judith Warner(12).

Dans le sillage du scandale de 2011 autour du "grand séducteur ", surnom donné à Dominique Strauss-Kahn, la responsable parisienne du bureau du New York Times, l'Américaine Elaine Sciolino, a écrit tout un livre sur le sujet(13), censé révéler " la vérité cachée sur le mode de vie français : tout est affaire de séduction (...). La France est un pays séducteur, séducteur par son élégance, sa beauté, ses plaisirs sensuels et sa joie de vivre(14). La séduction est bien plus qu'un jeu pour les Français : c'est la clé d'une bonne compréhension de la France. "

Pendant ce temps, la chroniqueuse politique canadienne anglophone Rachel Marsden titrait, à propos de ce surnom, "Excusez-moi, s'il vous plait, while I plunge my head into la toilette(15). " Le concept de séduction, qui fait partie intégrante de la conscience nationale dans la culture française, est donc à manipuler avec des pincettes quand on s'adresse à un public anglophone de naissance.

(12) J. Warner, The Seduction Myth: What the French still don't get about sex, Time magazine (17/05/2011).

(13) E. Sciolino, La Seduction: How the French play the Game of Life (St. Martin's Griffin, 2012) - La Séduction : Comment les Français jouent au jeu de la vie. Trad. Nicolas Véron (Presses de la Cité, 2012).

(14) En français dans le texte.

(15) " Excusez-moi, s'il vous plaît, je vais plonger la tête dans la cuvette des toilettes ". R. Marsden, Daily News, 18 mai 2011. 


\section{Que faut-il pour être un bon traducteur dans le domaine du luxe?}

Lorsqu'on " vend du rêve ", la traduction n'est pas qu'une simple affaire de mots ou même d'image : il faut traduire une ambiance et un sens sous-jacents. II faut être psychologue, s'adapter à l'état d'esprit de différents publics et comprendre comment attirer les lecteurs dans l'" univers " de la marque et sa promesse distinctive (certains produits, comme des liqueurs haut de gamme, cherchent à attirer les provinciaux anglais d'un certain âge aussi bien que les rappeurs américains des villes).

Une fois que j'ai une idée juste de ce que " promet " la marque, je réfléchis à ce que ces notions particulières, ou ce discours, ont d'attirant pour le public de la langue source. Quelles émotions éveillent-elles ? S'agit-il d'une complicité affectueuse ? De la liberté de prendre ses distances? De l'excitation du risque? D'une séduction sans limites?

En plaçant ces éléments en parallèle du « nouveau » public visé, je me concentre sur les attentes communes pour trouver les bonnes images dans la langue cible, afin d'évoquer les mêmes sensations tout en gardant à l'esprit les différences qui existent dans les attitudes et les références culturelles.

Par exemple, certaines publicités françaises vantent des parfums ou de la lingerie en utilisant des scénarios assez explicites de femmes qui cherchent à subjuguer sexuellement des hommes, ce qui peut être perçu par un public britannique ou américain comme sexiste ou dévalorisant. La traduction doit donc soit ruser en adoptant un angle légèrement différent, soit désamorcer la connotation potentiellement négative par une touche d'humour complice. Le bagage culturel du traducteur et son expérience personnelle de ce qu'est le luxe joueront également un rôle important pour l'aider à comprendre et à exploiter les sous-entendus dans les deux langues/ cultures.

\section{Problèmes spécifiques de la traduction rédactionnelle du français vers l'anglais}

En français, il existe un large fossé entre le style rédactionnel classique et la familiarité des publicités et des réseaux sociaux, plus proche du français parlé avec des tournures telles que "Tu viens pas ? " ou "Faut voir ça ! ". L'anglais a, pour sa part, développé un style écrit plus démocratique et largement accessible, ni trop guindé ni trop familier, mais plus passe-partout.

Pour déclencher des émotions, les professionnels français du marketing ont tendance à utiliser un registre de langue soutenu, volontairement complexe et opaque, tandis qu'en anglais on a besoin d'un langage plus direct afin de toucher les lecteurs à un niveau intuitif. En tant que traducteur, il peut être difficile de faire le grand écart et de conserver un ton élégant - sans 
être collet monté ni affecté - tout en écrivant dans un style fluide et contemporain, accessible sans être trop " populaire ". Le marché du luxe aime généralement se sentir en phase avec son temps, et parfois j'ai du mal à faire mon choix entre an über-minted clientèle et extremely wealthy clients pour désigner une clientèle très fortunée, c'est-à-dire à quel niveau me placer précisément.

Comme toute langue, le français a ses propres défis en traduction. Les textes rédactionnels, en particulier, et plus encore dans le domaine du luxe, nécessitent une compréhension très fine de la langue française. Ils se caractérisent par une grammaire relativement compliquée, avec des phrases interminables et de longues propositions relatives, séparées uniquement par des virgules, jusqu'à ce qu'on ait perdu le fil de la hiérarchie. Pour les anglophones, il y a un grand nombre de faux amis dont il faut se méfier (particulièrement dangereux lorsque la différence de sens est minime), sans oublier les adjectifs qui changent de sens selon qu'ils sont placés avant ou après un nom.

Parfois, j'aborde la première étape de la traduction comme une équation mathématique. Je décompose la phrase pour en extraire les divers arguments et en dresser la liste, afin de m'aider à associer chaque adjectif avec le(s) mot(s) correspondant(s), et à déterminer les relations qui lient les différentes propositions.

La plupart du temps, je traduis pour un public anglophone international, de sorte qu'il est souvent approprié de parsemer le texte de mots français pour lui conférer un peu de classe des expressions comme " par excellence ", " je ne sais quoi " et " panache " sont souvent " de rigueur " en anglais.

\section{Mon point de vue personnel}

J'ai toujours été fascinée par la psychologie et le comportement, et j'ai une expérience professionnelle dans la communication et la publicité : la traduction dans le domaine du luxe me convient à merveille. J'aime le charme de la beauté et du savoir-faire artisanal, d'une présentation et d'un service impeccables. En traduisant dans le domaine du luxe, je laisse libre cours à mon style d'écriture fluide et imaginatif.

J'ai la chance de travailler principalement du français vers l'anglais, puisque beaucoup de marques de luxe (peut-être même la majorité) ont leur siège en France ou en Suisse romande. Plus j'en apprends sur l'artisanat d'art, la joaillerie, la maroquinerie ou l'horlogerie, plus je me rends compte que j'aime ces sujets. J'adore décrire (et observer) des bijoux fabuleusement chers, des sacs à main magnifiquement ouvragés à partir de cuirs et peaux exotiques, des montres d'une finesse exquise. Lorsque j'ai commencé, je me souviens que les prix m'ont choquée ; mais au fur et à mesure que j'ai appris les détails sur la fabrication de ces objets et les compétences incroyables qu'ils nécessitent, j'ai commencé à comprendre leur "valeur ". 
Ma conscience de ce qui constitue un service impeccable et de ce qui n'est pas à la hauteur s'est clairement affinée. Je suis de plus en plus pointilleuse, ce qui n'est pas nécessairement une qualité...

\section{Immoral ou sublime : mais pour combien de temps encore ?}

Ce qui m'amène aux questions morales du travail dans le domaine du luxe. Je suis parfaitement consciente de l'injustice inhérente à ce secteur, des impacts négatifs du marketing contemporain qui nous encourage à être insatiables, et du caractère non durable d'une grande part du secteur du luxe - il y aurait beaucoup à dire à ce sujet. Lorsque je lis des articles sur l'austérité dans les journaux, j'ai l'impression de travailler dans un univers parallèle.

D'un certain point de vue, la production d'objets de luxe frôle l'immoralité. D'un autre côté, je pense que certains aspects de ce secteur sont l'expression des plus belles réalisations humaines, qui permettent à des savoir-faire incroyables de se perpétuer et donnent naissance à des objets fabuleux, comme les œufs commandés à Fabergé par les tsars de Russie (l'CEuf de l'hiver 1913 aurait coûté plus de 3 millions d'euros de nos jours).

Le lien entre le luxe et l'art a toujours été étroit, et aujourd'hui de nombreuses maisons de luxe tentent d'apaiser leur conscience sociale en remplissant les fonctions morales des riches, en pratiquant le mécénat et en redistribuant une partie de leurs gains par l'intermédiaire d'organismes caritatifs et de fondations. Elles se positionnent en nouveaux philanthropes, " noblesse oblige ", dirait-on en bon anglais.

Néanmoins, la météo n'est pas au beau fixe pour ce secteur. La croissance a nettement ralenti depuis début 2015 et la tendance devrait se poursuivre, entre troubles géopolitiques, changements des habitudes d'achat et nouvelle donne touristique. Qu'est-ce que l'avenir peut réserver à l'industrie du luxe?

Un professionnel du secteur, Nader Mousavizadeh(16), a un point de vue original : " Je crains que les produits de luxe ne soient pas tendance pour toute une génération de très fortunés". D’après lui, les jeunes consommateurs « assimileront les produits de luxe à l'inégalité croissante

(16) Nader Mousavizadeh, de l'agence de conseil en investissement Macro Advisory Partners, cité dans To have and have not: Inequality hits luxury, Kate Abnett, Business of Fashion (27/6/2016) - traduction publiée dans M, le magazine du Monde (01/8/2016). 
des revenus - à leurs yeux, un mal social et le principal enjeu pour leur génération. Hermès sera comparé à l'entreprise pétrolière Shell, non parce que ses produits sont toxiques, mais parce que ses clients sont les bénéficiaires de cette inégalité de revenus exacerbée ".

Cela dit, je crois que mon emploi n'est pas en danger pour l'instant.

helene@besoindanglais.com

Traduit de l'anglais par Marie-Céline Georg (et Christine Cross).

Helene Tammik. Luxurious translations.

(lauréate du concours 2015 " Traduire à Chantilly " - FR > EN ;

Jury : Chris Durban, Grant Hamilton, Ros Schwartz, François Lavallée, Dominique Jonkers \& Marc Lambert)

Helene Tammik est traductrice du français vers l'anglais et rédactrice en anglais, spécialisée dans les textes commerciaux et rédactionnels pour des clients très haut de gamme. Elle baigne dans le monde des mots et de la communication depuis plus de 25 ans. Sa carrière a commencé dans la publicité et le marketing à Londres, avant d'évoluer vers la traduction. Cette psychothérapeute diplômée possède également une longue expérience de l'enseignement de l'anglais des affaires à des cadres français et du coaching en communication. Avec ce riche bagage professionnel, elle peut apporter le meilleur de son expérience en rédaction marketing et créative à la communication des marques, et trouver exactement le ton juste pour s'adresser aux lecteurs anglophones du monde entier.

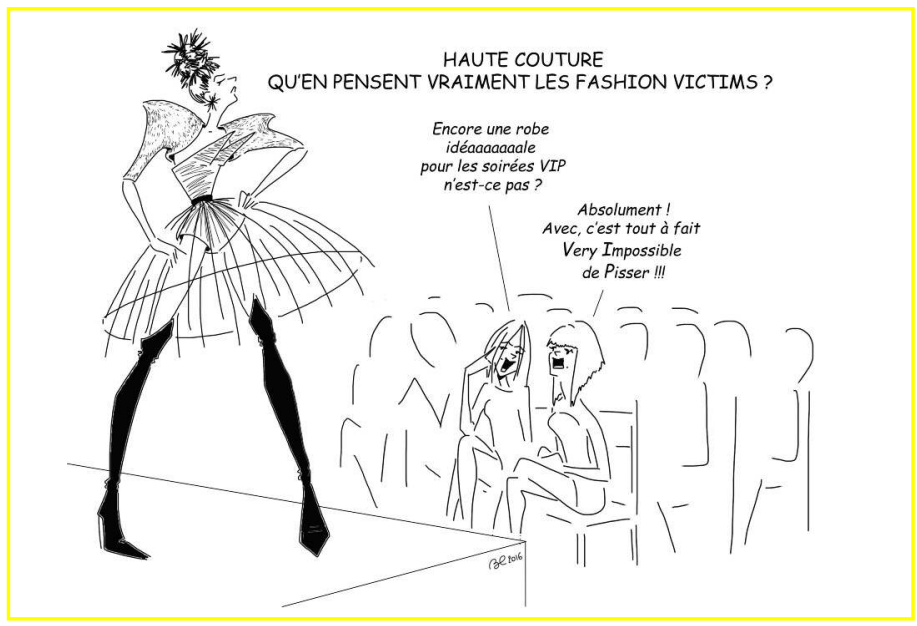

